\title{
Pathologic Risk Factors and Oncologic Outcomes in Early-stage Cervical Cancer Patients Treated by Radical Hysterectomy and Pelvic Lymphadenectomy at a Thai University Hospital: A 7 year Retrospective Review
}

\author{
Irene Ruengkhachorn ${ }^{1 *}$, Suwanit Therasakvichya ${ }^{1}$, Malee Warnnissorn ${ }^{2}$, Chairat \\ Leelaphatanadit $^{1}$, Suthi Sangkarat ${ }^{1}$, Jutatip Srisombat ${ }^{1}$
}

\begin{abstract}
Background: To evaluate the rate of pathologic high-risk factors, intermediate-risk factors, and treatment outcomes in early-stage cervical cancer patients undergoing radical hysterectomy and pelvic lymphadenectomy (RHPL). Materials and Methods: Medical records of stage IA-IIA1 cervical cancer patients who underwent RHPL during the 2006 to 2012 time period and patient follow-up data until December 2013 were reviewed. Results: Of 331 patients, 52 women $(15.7 \%)$ had pathologic high-risk factors and 59 women $(17.8 \%)$ had intermediate-risk factors without high-risk factors. All studied patients had an initial complete response. At median follow-up time of 40.9 months (range 1-103.3 months) and mean follow-up time of $43.3 \pm 25.3$ months, 37 women had disease recurrence and 4 women had died of disease. The most common site of recurrence was the pelvis $(64.8 \%)$. Fiveyear and 10-year disease free survival rates were $96.1 \%$ and $91.5 \%$, respectively. Five-year and 10-year overall survival rates were $100 \%$ and $99.4 \%$, respectively. Independent factors related to recurrence were pelvic node metastasis (odds ratio [OR], 2.670; 95\% CI, 1.001-7.119), and $>1 / 3$ cervical stromal invasion $(\mathrm{OR}, 3.763$; $95 \% \mathrm{CI}$, 1.483-9.549). Conclusions: The rates of pathologic high-risk and intermediate-risk factors should be considered and disclosed when counseling patients regarding primary treatment by RHPL. Oncologic outcomes of primary surgical treatment for early-stage cervical carcinoma were found to be excellent.
\end{abstract}

Keywords: Pathologic risk factors - cervix - cervical cancer - radical hysterectomy

Asian Pac J Cancer Prev, 16 (14), 5951-5956

\section{Introduction}

Cervical carcinoma is the most common gynecologic cancer and the second most common cancer in Thai women with an age standardized rate (ASR) of 16.7 per 100,000 populations (Attasara and Sriplung, 2013). Comparison between early-stage cervical cancer patients who underwent either radiation or surgery as primary therapy showed 5-year survival rates of $80-90 \%$ in both groups (Newton, 1975; Landoni et al., 1997). Radical surgery offers superior benefit in tumor removal, precise evaluation of spread of disease, preservation of vaginal length and elasticity, and retention of ovarian function. Adjuvant treatment and disease outcomes depend on pathologic risk factors. Patients with at least one pathologic high-risk factor, including positive surgical margins, parametrium (PRM) invasion, or pelvic lymph node metastasis, was found the 5-year survival rate was decreased and the risk of disease recurrence was increased to $40 \%$ (Peters et al., 2000). A study by the Gynecologic Oncology Group (GOG) reported that patients presenting with pathologic intermediate-risk factors, such as large tumor size, deep stromal invasion (DSI), or lymphovascular space invasion (LVSI), had a risk of recurrence as high as 30\% (Sedlis et al., 1999; Rotman et al., 2006).

The objectives of this study were to (i) analyze type and incidence rates of pathologic risk factors, and (ii) evaluate disease outcomes in patients with stage IA-IIA1 cervical cancer, for whom primary treatment was radical hysterectomy and pelvic lymphadenectomy (RHPL).

\section{Materials and Methods}

The study protocol was approved by the Siriraj Institution Review Broad (COA no. Si463/2013). Medical records from 331 stage IA-IIA cervical cancer patients who were schedule for RHND in Siriraj Hospital, from 1 
January 2006 to 31 December 2012 were retrospectively reviewed. Patient data retrieved included baseline data, clinical staging, surgical procedures, histopathology, adjuvant treatment, and outcomes.

Clinical staging was determined by one radiation oncologist and one gynecologic oncologist at the hospital's tumor registry unit, according to the International Federation of Gynecology and Obstetrics (FIGO) recommendations (Benedet et al., 2000; Pecorelli et al., 2009). Surgical treatment procedures were scheduled within 6 weeks of clinical staging date. In patients confirmed by frozen section to have disease metastasis outside the uterus (pelvic lymph node or PRM) the plan for treatment by hysterectomy was abandoned. PRM involvement was defined as tumor invasion of parametrial tissue and/or parametrial nodes. Large tumor size was defined as clinical tumor size $\geq 4 \mathrm{~cm}$. DSI was defined as carcinoma invasion into the middle or deep third of the total cervical stromal thickness. LVSI was defined as tumor invasion into the endothelium of vascular and/or lymphatic vessels. Patients with at least one pathologic high-risk factor were advised to consider for adjuvant concurrent chemoradiation, according to GOG 109 (Peters et al., 2000). In patients who presented with two or more intermediate-risk factors but no pathologic highrisk factors, adjuvant radiation alone was recommended. Disease free survival (DFS) time was defined as date of surgery to date of clinical or radiographic evidence of disease recurrence or until date of last follow-up, in cases of no disease recurrence. Overall survival (OS) time was defined as date of surgery to date of death or date of last contact.

Statistical analysis was performed with SPSS software

Table 1. Baseline Characteristics and Clinical Staging from 331 Patients

\begin{tabular}{lr}
\hline Characteristics & \multicolumn{1}{c}{$\mathrm{n}(\%)$} \\
\hline Parity $\geq 1$ & $294(88.8)$ \\
Postmenopause & $125(37.8)$ \\
Body mass index classification & \\
Underweight $(<18.5 \mathrm{~kg} / \mathrm{m} 2)$ & $21(6.3)$ \\
Normal weight $(18.5-24.9 \mathrm{~kg} / \mathrm{m} 2)$ & $180(54.4)$ \\
Overweight $(25.0-29.9 \mathrm{~kg} / \mathrm{m} 2)$ & $95(28.7)$ \\
Obesity $(\geq 30.0 \mathrm{~kg} / \mathrm{m} 2)$ & $35(10.6)$ \\
Presenting symptoms & \\
Check-up & $169(51.1)$ \\
Vaginal bleeding & $127(38.4)$ \\
Discharge & $27(8.2)$ \\
Pelvic pain & $9(2.1)$ \\
Histopathology & \\
Squamous cell carcinoma & $192(58.0)$ \\
Adenocarcinoma & $113(34.1)$ \\
Adenosquamous & $15(4.6)$ \\
Mixed type & $5(1.5)$ \\
Other & $6(1.8)$ \\
FIGO stage & $34(10.3)$ \\
IA1 & $3(0.9)$ \\
IA2 1 IB2 & \\
IIA1 & $38(11.5)$ \\
\hline
\end{tabular}

*FIGO, the International federation of gynecology and obstetrics version 21.0 (IBM, Armonk, NY, USA). Baseline characteristics, rates of pathologic high-risk factors, rates of $\geq 2$ intermediate-risk factors without pathologic high-risk factors, patterns of disease spread, and disease outcomes were analyzed using descriptive statistics. Various factors associated with recurrence were analyzed by regression analysis and described as odds ratio (OR) and $95 \%$ confidence interval (CI). Survival outcome curves were created using the Kaplan-Meier method.

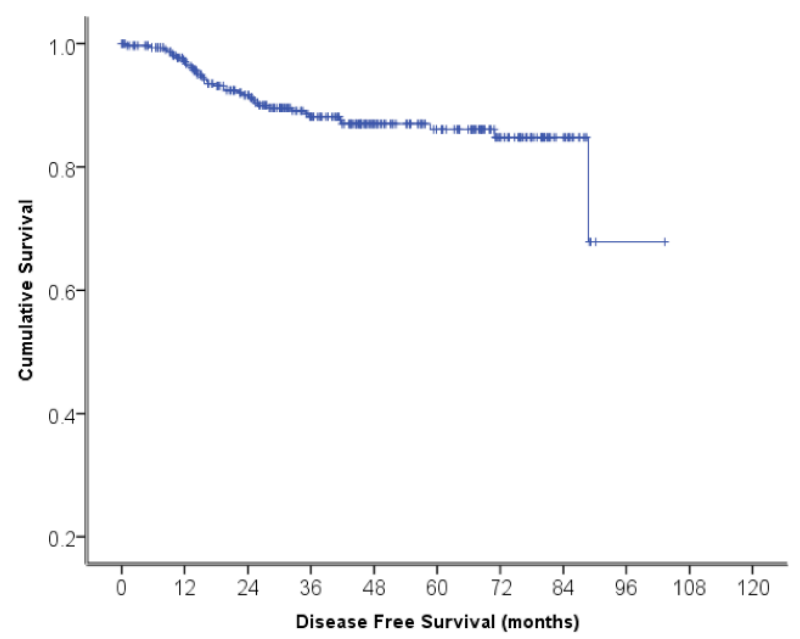

Figure 1. Progression of Disease-Free Survival from 331 Patients

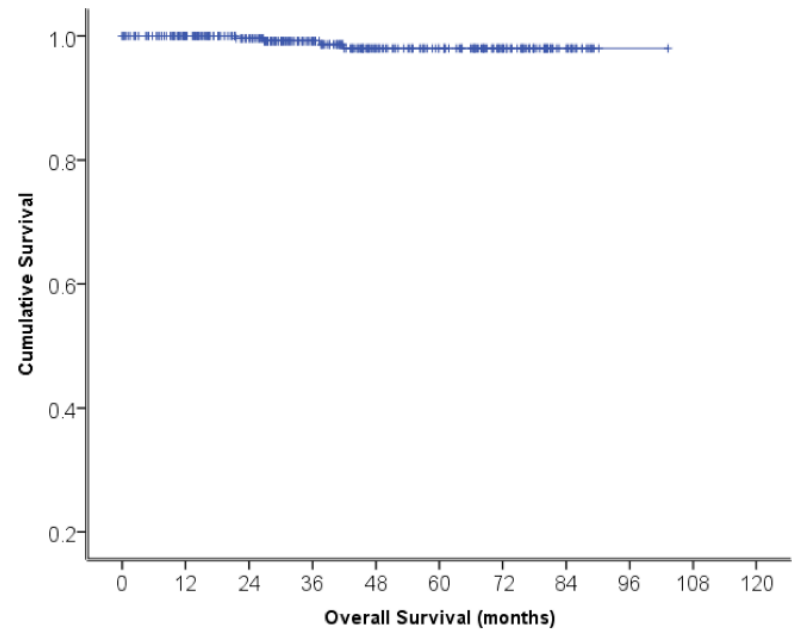

Figure 2. Overall Survival from 331 Patients

Table 2. Rates of Pathologic High and IntermediateRisk Factors from 331 Patients

\begin{tabular}{lr}
\hline Pathologic risk factors & $\mathrm{n}(\%)$ \\
\hline $\begin{array}{l}\text { No pathologic risk factors, } \mathrm{n}=331 \\
\text { Pathologic high-risk factors }\end{array}$ & $220(66.5)$ \\
Positive surgical margins, $\mathrm{n}=323$ & $17(5.3)$ \\
Parametrial involvement, $\mathrm{n}=325$ & $20(6.2)$ \\
Pelvic lymph node metastasis, $\mathrm{n}=331$ & $33(9.9)$ \\
Pathologic intermediate-risk factors & \\
Clinical tumor size $\geq 4 \mathrm{~cm}, \mathrm{n}=331$ & $28(8.5)$ \\
DSI, $\mathrm{n}=323$ & $175(52.9)$ \\
LVSI, $\mathrm{n}=323$ & $106(32.0)$ \\
\hline *DSI, deep stromal invasion; LVSI, lymph vascular space invasion
\end{tabular}

*DSI, deep stromal invasion; LVSI, lymph vascular space invasion 


\section{Results}

From the 7-year timeframe of this study, a total of 331 patient records met the study criteria. Patient demographic and preoperative clinical data was demonstrated in Table 1. Mean age and median age were $48.6 \pm 10.1$ years and 48.0 years (range 25-78 years), respectively. The range of parity was $0-10$, with a mean of $2.3 \pm 1.5$ and a median of 2.0. Mean body mass index (BMI) was $24.4 \pm 4.3 \mathrm{~kg} /$ $\mathrm{m}^{2}$, with median of $23.7 \mathrm{~kg} / \mathrm{m}^{2}$ (range $14.8-40.6 \mathrm{~kg} / \mathrm{m}^{2}$ ). Diagnostic methods were; punch biopsy in 147 patients $(44.4 \%)$, colposcopic-directed biopsy in 48 patients $(14.5 \%)$, curettage in 14 patients $(4.2 \%)$, and conization in 121 patients $(36.9 \%)$.

Surgical approached was laparoscopy in 54 patients $(16.3 \%)$. Of 331 operations, mean operative time was $255 \pm 64$ minutes, with median of 247 minutes (range 60 570 minutes). Mean blood loss was $657 \pm 560 \mathrm{~mL}$ with median of $500 \mathrm{~mL}$ (range 20-4,000 mL). Of 331 patients, 8 patients $(2.4 \%)$ resulted in abandoned hysterectomy. Rationale for abandonment described, as follows: adnexal metastasis (1 patient), severe pelvic endometriosis (1 patient), pelvic node metastasis (4 patients), PRM metastasis (1 patient), and PRM and pelvic node metastasis (1 patient). Intra-operative adjacent organ injuries were found in 9 patients $(2.7 \%)$, as follows; ureter or bladder injury ( 7 patients), obturator nerve injury (1 patient), and lumbosacral nerve injury (1 patient). Seventy patients (21.1\%) had operative blood loss in excess of 1,000 mL for laparotomy or $500 \mathrm{~mL}$ for laparoscopy, and 37 of those patients required blood transfusion. Surgical site infection (SSI) developed in 9 patients $(2.7 \%)$.

Final pathologic results showed no residual tumor in 48 patients (14.5\%), and residual high-grade squamous intraepithelial lesion (HSIL) or adenocarcinoma in situ (AIS) in 22 patients $(6.6 \%)$. Regarding the pathologic risk factors described in Table 2, 52 patients $(15.7 \%)$ had at least one pathologic high-risk factor and 59 patients (17.8\%) had $\geq 2$ intermediate-risk factors without highrisk factors. Moreover, 97 patients $(29.3 \%)$ received adjuvant radiation therapy, with 62 patients treated by concurrent chemo-irradiation and 35 patients treated by

Table 3. Variables Correlated with Recurrence of Disease from 331 Patients

\begin{tabular}{|c|c|c|c|}
\hline Variables & Rate of recurrence $(\%)$ & $\begin{array}{c}\text { Univariate } \\
\text { OR }(95 \% \mathrm{CI}), \mathrm{p} \text {-value }\end{array}$ & $\begin{array}{c}\text { Multivariate } \\
\text { OR }(95 \% \mathrm{CI}), \mathrm{p} \text {-value }\end{array}$ \\
\hline \multicolumn{4}{|c|}{ Hemoglobin (g/dL), n=331 } \\
\hline$\geq 12.5$ & $11 / 137(8.0)$ & $0.564(0.269-1.184), 0.127$ & \\
\hline$<12.5$ & 26/194 (13.4) & & \\
\hline \multicolumn{4}{|l|}{ Obesity, $n=331$} \\
\hline Yes & $14 / 130(10.8)$ & $0.934(0.462-1.889), 0.849$ & \\
\hline No & 23/201 (11.4) & & \\
\hline \multicolumn{4}{|c|}{ Histology, $n=331$} \\
\hline Non-SCCA & $18 / 139(12.9)$ & $1.355(0.683-2.688), 0.384$ & \\
\hline SCCA & 19/192 (9.9) & & \\
\hline \multicolumn{4}{|c|}{ FIGO stage, $n=331$} \\
\hline IA-IB 1 & $4 / 72(5.6)$ & $2.482(0.849-7.255), 0.087$ & \\
\hline IB2-IIA1 & $33 / 259(12.7)$ & & \\
\hline \multicolumn{4}{|c|}{ Pelvic nodes metastasis, $\mathrm{n}=331$} \\
\hline Yes & $11 / 33(33.3)$ & $5.231(2.285-11.973),<0.001$ & $2.670(1.001-7.119), 0.05$ \\
\hline No & $26 / 298(8.87)$ & & \\
\hline \multicolumn{4}{|c|}{ Parametrial metastasis, $\mathrm{n}=325$} \\
\hline Yes & $2 / 20(10.0)$ & $0.928(0.218-4.434), 0.669$ & \\
\hline No & $31 / 305(10.2)$ & & \\
\hline \multicolumn{4}{|c|}{ Surgical margin involvement, $\mathrm{n}=323$} \\
\hline Yes & $1 / 17(5.9)$ & $0.535(0.069-4.170), 1.0$ & \\
\hline No & $32 / 306(10.5)$ & & \\
\hline \multicolumn{4}{|c|}{ Uterine metastasis, $\mathrm{n}=323$} \\
\hline Yes & $4 / 25(16.0)$ & $1.767(0.567-5.501), 0.304$ & \\
\hline No & 29/298 (9.7) & & \\
\hline \multicolumn{4}{|c|}{ Vaginal metastasis, $\mathrm{n}=323$} \\
\hline Yes & $3 / 12(25.0)$ & $3.122(0.802-12.162), 0.113$ & \\
\hline No & $30 / 311(9.6)$ & & \\
\hline \multicolumn{4}{|l|}{ DSI, $n=323$} \\
\hline Yes & $27 / 175(15.4)$ & $4.318(1.731-10.770), 0.001$ & $3.763(1.483-9.549), 0.005$ \\
\hline No & $6 / 148(4.1)$ & & \\
\hline \multicolumn{4}{|l|}{ LVSI, $n=323$} \\
\hline Yes & $14 / 106(13.2)$ & $1.586(0.762-3.302), 0.215$ & \\
\hline No & $19 / 217(8.8)$ & & \\
\hline \multicolumn{4}{|c|}{ Pathologic HR/IMR factors, $n=331$} \\
\hline Yes & $18 / 111(16.2)$ & $2.048(1.027-4.082), 0.039$ & \\
\hline No & $19 / 220(8.6)$ & & \\
\hline
\end{tabular}


radiation alone.

All of the patients evaluated in this study had initial complete response. At median follow-up time of 40.9 months (range 1-103.3 months) with a mean of $43.3 \pm 25.3$ months, 37 women (11.2\%) had disease recurrence and 4 women $(1.2 \%)$ died of their disease. The most common site of recurrence was the pelvis, 24 women of 37 women $(64.8 \%)$, follow by distant metastasis (12 women, $32.4 \%$ ), and one women had both locoregional and distant recurrence. 306 women (92.4\%) alive without disease on the last contact date. Variables associated with disease recurrence were described in Table 3. Figure 1 showed DFS of studied patients with a mean of $89.2 \pm 2.8$ months (95\%CI 83.7-94.7). The 5-year and 10-year DFS rates were $96.1 \%$ and $91.5 \%$, respectively. Rate of OS was $100 \%$ at 5 -years and $99.4 \%$ at 10 -years (Figure 2). The mean OS duration was $101.9 \pm 0.7$ months $(95 \% \mathrm{CI}$ 100.5-103.2).

\section{Discussion}

Radical surgery, the standard treatment option for early-stage cervical carcinoma, has a high cure rate as compared to that of primary radiotherapy (Landoni et al., 1997). RHPL is considered in FIGO stages IA1 or IA2 when invasive disease is identified at cone margins or when clinicians are not confident in the pathological evaluation of depth invasion in non-squamous cell carcinoma (non-SCCA) histology, given-the inherently unpredictable infiltrative behavior of non-SCCA.

Substantially, we found that $33.2 \%$ of RHPL patients at Siriraj Hospital required adjuvant radiation therapy. The percentage of patients who presented with at least one of pathologic high-risk factor was $15.7 \%$, as follows; PRM metastasis $(6.2 \%)$, pelvic node involvement $(9.9 \%)$ and disease at surgical margins $(5.3 \%)$. From our previous study, rates of pelvic node metastasis and PRM metastasis were $0 \%$ and $4.5 \%$ in stage IA1, respectively, and $6.1 \%$ and $9.1 \%$ in stage IA2, respectively (Chatchotikawong et al., 2014). McCann et al., (2013) studied stage IA2IIA patients and found pelvic node metastasis and PRM metastasis rates of $34 \%$ and $22 \%$, respectively. From an Italian study in stage IA2-IIA cervical carcinoma, rate of pelvic node metastasis was $16.3 \%$ (Ghezzi et al., 2007). Siu et al., (2006) reported a pelvic node metastasis rate of $20.1 \%$ in stage IB-IIA patients. Landoni et al., (2001) reported rates of $24.8 \%$ for pelvic node metastasis and $26.1 \%$ for PRM involvement in stage IB-IIA patients. A Korean study reported a stage IB1 nodes metastasis rate of $16.5 \%$ (Lee et al., 2007). In stage IB1-IB2 patients, a previous study revealed pelvic node metastasis and PRM metastasis rates of $12.8 \%$ and $10.1 \%$, respectively (Rutledge et al., 2004). Covens et al., (2002) studied 842 stage IA1-IB1 cervical carcinoma patients who underwent RHPL and found rates of pelvic node metastasis of $6 \%$ and PRM metastasis of $4 \%$. Patients with stage IA2-IB1 cervical carcinoma had equal rate of pelvic nodal and PRM metastasis rates at 7.5\% (Ditto et al., 2015). The disparity in the rate of pathologic high-risk factors in patients with early-stage cervical carcinoma is a consequence of heterogeneity in patient characteristics, including; tumor stages, diversity of human papilloma virus (HPV) typing, and race.

In this study, the percentage of patients with $\geq 2$ intermediate-risk factors, but no high-risk factors was $17.8 \%$. This rate was inconsistent with previous reported findings, due to possible discrepancies in intermediaterisk criteria. From the GOG 92 study of stage IB patients, rate of intermediate-risk factors was $13 \%$. The GOG intermediate-risk factor criteria satisfied one of following; (i) positive LVSI and deep third cervical stromal invasion, (ii) positive LVSI, middle or deep third invasion, and clinical tumor size $\geq 2 \mathrm{~cm}$, (iii) positive LVSI, superficial third invasion and tumor size $\geq 5 \mathrm{~cm}$, or (iv) middle or deep third invasion and tumor size $\geq 4 \mathrm{~cm}$ (Sedlis et al., 1999). The Korean Gynecologic Oncology Group considers any two of four variables (tumor size $\geq 3 \mathrm{~cm}$, outer third of the cervical stromal invasion, positive LVSI, and adenocarcinoma or adenosquamous carcinoma histology), to be intermediate-risk factors. The Korean study found that $63.8 \%$ of stage IB-IIA patients met their intermediate-risk criteria; whereas, only $36.1 \%$ of patients met the GOG criteria (Ryu et al., 2014).

From our study, the $11.2 \%$ recurrent rate and $1.2 \%$ disease-related death rate were comparable with previous studies (Ghezzi et al., 2007; McCann et al., 2013). From a study of stage IB-IIA patients by Landoni et al., (2001) $25.2 \%$ experienced disease recurrence and $18.9 \%$ died from disease after being treated by class II or III radical hysterectomy. In the present study, independent factors that significantly associated with disease recurrence were pelvic node metastasis and DSI. All of 37 recurrent women, 11 women had previous report of pelvic node metastasis. Of which, 4 women had locoregional and 7 women had distant recurrence. Patients with pelvic node metastasis were prescribed adjuvant CCRT, consistent with standard clinical recommendations. Accordingly, in patients with pelvic node metastasis, the authors recommend considering additional adjuvant systemic chemotherapy after complete adjuvant CCRT.

While standards and protocols may vary by country, DSI alone is not generally considered an intermediate-risk criterion that requires adjuvant radiation. For our institute, the authors think that DSI alone might be considering for adjuvant radiation. However, this need future prospective study for verify. Furthermore, future research in the areas of genomics and epigenetics will help to more accurately identify factors that correlate with disease recurrence. This diagnostic advancement will supplement and perhaps replace our current method of relying on pathologic factors alone. Human papillomavirus (HPV) status and expression of selected markers, such as HER2/neu, epidermal growth factor receptor (EGFR), vascular endothelial growth factor (VEGF), CD34, p63, p53, Ki67/MIB-1, bcl-2, major vault protein (MVP) and insulin-like growth factor receptor-1 (IGF-1R) expression may be found to be more efficacious than pathologic evaluation in identifying patients at highrisk for disease recurrence (Henríquez-Hernández et al., 2011; Vosmik et al., 2014).

Although there was some debate regarding histology typing, non-SCCA was not associated with poor prognosis in our study, as reported in some previous studies (Takeda 
et al., 2002; Ditto et al., 2015). However, there was a study in stage IB patients revealed higher recurrence rate in non-SCCA type (Srisomboon et al., 2011). The stage of the disease may have a great impact on the prognosis of non-SCCA type.

Landoni et al., (2001) reported ureteric fistula and nerve injury after RHPL, 1 patient $(0.9 \%)$ each. Another study of 201 RHPL patients reported ureteric injury $(1.5 \%)$, urinary tract fistula $(0.5 \%)$, and SSI $(4.0 \%)$ (Pikaart et al., 2007). A study of RHPL in 480 patients revealed rates of ureteric injury of $0.8 \%$, bladder injury of $0.8 \%$, urinary tract fistula of $1.0 \%$, and SSI of $0.6 \%$ (Cai et al., 2009). The National Health Service (NHS) study in 343,771 hysterectomy procedures, revealed a urinary fistula rate of 1 in 788, with the highest rate found in RHPL 1 in 87 with $95 \%$ CI of 61-128 (Hilton and Cromwell, 2012). The current study, urinary tract injury rate was 5 in 323 RHPL patients $(1.5 \%)$ and urinary tract fistula rate was 2 in 323 RHPL patients $(0.6 \%)$. These rates were comparable to the results reported in previous studies.

Strengths of this study include: single institution uniform surgical technique, representative data from a country with high incidence of cervical carcinoma, substantial sample size with long-term follow-up period, and histopathology specimens evaluated by gynecologic pathologists. Regarding potential shortcomings, our study did not compare Siriraj Hospital criteria for intermediaterisk factors with GOG criteria for treatment complications and prediction of oncologic outcomes.

In conclusion, the rates of pathologic risk factors for recurrence after RHPL were rather high. Clinicians should consider and weigh long-term complications, especially in cases of combined radical surgery and adjuvant radiation therapy. Oncologic outcomes of primary surgical treatment for early-stage cervical carcinoma were found to be excellent.

\section{Acknowledgements}

The authors gratefully acknowledge Dr. Saowalak Hunnangkul, Epidemiology Unit, Siriraj Hospital, for statistical support. This study was supported by a grant from the Siriraj Research Development Fund (R015631045).

\section{References}

Attasara P, Sriplung H (2013). Cancer incidence in Thailand. In: Khuhaprema T, Attasara P, Sriplung H, Wiangnon S, Sangrajrang S, eds. Cancer in Thailand Vol. VII. Bangkok: Lyon: International Agency for research on Cancer, 8-76.

Benedet JL, Bender H, Jones H, 3rd, Ngan HY, Pecorelli S (2000). FIGO staging classifications and clinical practice guidelines in the management of gynecologic cancers. FIGO Committee on Gynecologic Oncology. Int J Gynaecol Obstet, 70, 209-62.

Cai HB, Chen HZ, Zhou YF, Lie DM, Hou HY (2009). Class II radical hysterectomy in low-risk IB squamous cell carcinoma of cervix: a safe and effective option. Int J Gynecol Cancer, 19, 46-9.

Chatchotikawong U, Ruengkhachorn I, Leelaphatanadit C (2014). Residual disease following conization of women with stage IA-IB1 cervical carcinoma in a high incidence region. Asian Pac J Cancer Prev, 15, 7383-7.

Covens A, Rosen B, Murphy J, et al (2002). How important is removal of the parametrium at surgery for carcinoma of the cervix? Gynecol Oncol, 84, 145-9.

Ditto A, Martinelli F, Bogani G, et al (2015). Implementation of laparoscopic approach for type B radical hysterectomy: a comparison with open surgical operations. Eur J Surg Oncol, 41, 34-9.

Ghezzi F, Cromi A, Ciravolo G, et al (2007). Surgicopathologic outcome of laparoscopic versus open radical hysterectomy. Gynecol Oncol, 106, 502-6.

Henríquez-Hernández LA, Lloret M, Pinar B, et al (2011). BCL-2, in combination with MVP and IGF-1R expression, improves prediction of clinical outcome in complete response cervical carcinoma patients treated by radiochemotherapy. Gynecol Oncol, 122, 585-9.

Hilton P, Cromwell DA (2012). The risk of vesicovaginal and urethrovaginal fistula after hysterectomy performed in the English National Health Service--a retrospective cohort study examining patterns of care between 2000 and 2008. BJOG, 119, 1447-54.

Landoni F, Maneo A, Colombo A, et al (1997). Randomised study of radical surgery versus radiotherapy for stage Ib-IIa cervical cancer. Lancet, 350, 535-40.

Landoni F, Maneo A, Cormio G, et al (2001). Class II versus class III radical hysterectomy in stage IB-IIA cervical cancer: a prospective randomized study. Gynecol Oncol, 80, 3-12.

Lee JM, Lee KB, Lee SK, Park CY (2007). Pattern of lymph node metastasis and the optimal extent of pelvic lymphadenectomy in FIGO stage IB cervical cancer. J Obstet Gynaecol Res, 33, 288-93.

McCann GA, Taege SK, Boutsicaris CE, et al (2013). The impact of close surgical margins after radical hysterectomy for early-stage cervical cancer. Gynecol Oncol, 128, 44-8.

Newton M (1975). Radical hysterectomy or radiotherapy for stage I cervical cancer. A prospective comparison with 5 and 10 years follow-up. Am J Obstet Gynecol, 123, 535-42.

Pecorelli S, Zigliani L, Odicino F (2009). Revised FIGO staging for carcinoma of the cervix. Int J Gynaecol Obstet, 105, 107-8.

Peters WA, 3rd, Liu PY, Barrett RJ, 2nd, et al (2000). Concurrent chemotherapy and pelvic radiation therapy compared with pelvic radiation therapy alone as adjuvant therapy after radical surgery in high-risk early-stage cancer of the cervix. $J$ Clin Oncol, 18, 1606-13.

Pikaart DP, Holloway RW, Ahmad S, et al (2007). Clinicalpathologic and morbidity analyses of Types 2 and 3 abdominal radical hysterectomy for cervical cancer. Gynecol Oncol, 107, 205-10.

Rotman M, Sedlis A, Piedmonte MR, et al (2006). A phase III randomized trial of postoperative pelvic irradiation in Stage IB cervical carcinoma with poor prognostic features: followup of a gynecologic oncology group study. Int J Radiat Oncol Biol Phys, 65, 169-76.

Rutledge TL, Kamelle SA, Tillmanns TD, et al (2004). A comparison of stages IB1 and IB2 cervical cancers treated with radical hysterectomy. Is size the real difference? Gynecol Oncol, 95, 70-6.

Ryu SY, Kim MH, Nam BH, et al (2014). Intermediate-risk grouping of cervical cancer patients treated with radical hysterectomy: a Korean gynecologic oncology group study. Br J Cancer, 110, 278-85.

Sedlis A, Bundy BN, Rotman MZ, et al (1999). A randomized trial of pelvic radiation therapy versus no further therapy in selected patients with stage IB carcinoma of the cervix after radical hysterectomy and pelvic lymphadenectomy: 
A gynecologic oncology group study. Gynecol Oncol, 73, 177-83.

Siu SS, Cheung TH, Lo KW, Yim SF, Chung TK (2006). Is common iliac lymph node dissection necessary in early stage cervical carcinoma? Gynecol Oncol, 103, 58-61.

Srisomboon J, Kietpeerakool C, Suprasert P, et al (2011). Survival and prognostic factors comparing stage IB 1 versus stage IB 2 cervical cancer treated with primary radical hysterectomy. Asian Pac J Cancer Prev, 12, 1753-6.

Takeda N, Sakuragi N, Takeda M, et al (2002). Multivariate analysis of histopathologic prognostic factors for invasive cervical cancer treated with radical hysterectomy and

systematic retroperitoneal lymphadenectomy. Acta Obstet Gynecol Scand, 81, 1144-51.

Vosmik M, Laco J, Sirak I, et al (2014). Prognostic significance of human papillomavirus (HPV) status and expression of selected markers (HER2/neu, EGFR, VEGF, CD34, p63,p53 and Ki67/MIB-1) on outcome after (chemo-) radiotherapy in patients with squamous cell carcinoma of uterine cervix. Pathol Oncol Res, 20, 131-7. 